\title{
Advances in Adenomyosis Diagnosis Utilizing Transvaginal Ultrasonography-A Short Summary
}

\author{
Kulvinder Kochar Kaur ${ }^{1 *}$, Gautam Allahbadia ${ }^{2}$ and Mandeep Singh ${ }^{3}$ \\ ${ }^{1}$ Department of Human Reproduction, India \\ ${ }^{2}$ Department of Obstetrics \& Gynecology, India \\ ${ }^{3}$ Department of Neurology, India \\ *Corresponding author: Kulvinder Kochar Kaur, Scientific Director, Centre of Human Reproduction, 721, G.T.B. Nagar, \\ Jalandhar-144001, Punjab, India
}

\section{Short Communication}

Adenomyosis is a frequent condition, being present in $20 \%$ of general gynae population [1,2] and $30-40 \%$ of those attending assisted reproductive technology clinics, having a detrimental effect on cases of in vitro fertilization (IVF) [3,4]. Its main characteristics are presence of heterotopic endometrial glands and stroma within the myometrium, $>2.5 \mathrm{~mm}$ in depth of myometrium or more than one microscopic field at 10times magnification from the endometrium-myometrium junction, along with a variable degree of adjacent myometrial hyperplasia, causing globular and cystic enlargement of the myometrium, with some cysts filled with extravasated, hemolyzed red blood cells and siderophages [5]. Till now the main belief was that this could only get diagnosed with the use of histology only. As this disease gets commonly encountered in women over $40 \mathrm{yrs}$, a belief got created that it was not a real disease. With the use of some imaging techniques like the magnetic resonance imaging and transvaginal ultrasound (TVS), it was found that adenomyosis had a typical appearance, which could be also detected in younger women, in the presence of symptoms or without, where it was found to have a typical appearance [6].

TVS is done in women of all ages and it showed typical sonographic findings, Different ultrasound imaging studies have been done to examine the diagnostic accuracy for finding adenomyosis as compared to that of histological examination of hysterectomy specimens. Also, the correlation of symptoms has been done. Biggest problem of using histology for adenomyosis diagnosis remains the big selection bias that has been observed. It had been seen that patients who had hysterectomy were usually in an advanced age and revealed heavy symptoms justifying surgery and thus do not represent the normal population. When a diagnosis of diffuse adenomyosis was done using TVS in younger fertile women who were with or without pain symptoms, a histological confirmation was found very occasionally of adenomyosis [7].

Inspite of this recently Tellum et al [8] tried to find the accuracy of TVS in diagnosis of adenomyosis. Using both 2D and 3D TVS, along with clinical symptoms, confirmed by histopathological examination they gave a predictive model, which showed a good test quality (area under curve $[\mathrm{AUC}]=0.86[95 \%$ confidence interval=0.79-0.94], optimal cutoff 0.56 , sensitivity of $85 \%$, specificity78\%). These 9 predictors were included ([sensitivity, specificity, $\beta]$ or [AUC $\beta]$; presence of myometrial cysts $(51 \%, 86 \%$, $\beta=0.86)$, fan shaped echo $(36 \%, 92 \%, \beta=0.54)$, hyperechoic islets $(51 \%, 78 \%, \beta=0.62)$, globular uterus $(61 \%, 83 \%, \beta=0.2)$, normal uterine shape $(83 \%, 61 \%, \beta=-0.75)$, thickest to thinnest ratio for uterine wall $(0.61, \beta=0.26)$, maximum width of the junctional zone in sagittal plane $(0.71, \beta=0.1)$, regular appearance of junctional zone $(31 \%, 92 \%, \beta=-1.0)$ and grade of dysmenorrhea measured on a verbal numerical scaling $(0.61, \beta=0.08)$. In view of various other studies, including that of Tellum et al. [8] showing a high accuracy of diagnosis, one can accept that one can make the diagnosis of adenomyosis just using ultrasonography (USG). One will be able to correlate the disease to real symptoms and fertility in the general population. Presence of one or more of USG features has often been observed in asymptomatic young ladies. 
The big problem encountered in this is that studies published till now on TVS and adenomyosis do not clearly represent how many features need to be considered for the final diagnosis of adenomyosis, since the prevalence of disease is in homogenous study populations. Yet TVS not only finds different features but can also give different configurations and localization inside the uterus as per the different histopathological adenomyosis types in the myometrium, namely the diffuse, focal and adenomyoma [9]. Important is not the number of features for finding adenomyosis since different features might be present in small focal adenomyosis and little features in a diffuse disease. Further the localization as per inner myometrium (junctional zone), or middle or outer myometrium, as per the degree of myometrium involvement may be important in describing adenomyosis by USG [10].

Since it is so like fibroids, adenomyosis must be described better inside to uterus for evaluating the impact on symptoms especially of infertility and treatment and utilize CA125 for differentiating the 2 [11]. Tellum et al [8] studies in certain ways like several features and thickness of the wall in their predictive model, confirmed that number of single USG features was not enough for providing an accurate diagnosis of adenomyosis .Still their predictive model can't be used in the general population as age, fertility, association to deep infiltrating endometriosis, the type (focal, diffuse) and the extension inside the myometrium of the adenomyosis have not been considered. Further, the correlation to pain symptoms correlating with menorrhagia in this study is to be queried as they included all patients who had surgery (mostly for symptomatic indications), of which $48 \%$ of patients having adenomyosis also had endometriosis, both can't cause similar symptoms.

Thus, although relevant one can use TVS for the diagnosis of adenomyosis, in view of it being an accurate and easy along with cheaper method that can be done on all types of patients. Presence of just a single TVS feature is not enough for the diagnosis of adenomyosis. Also, the correlation of presence or absence of symptoms to adenomyosis appears very superficial.
Just as endometriotic disease where ovarian, retroperitoneal and superficial disease had different effects in symptoms, treatment, prognosis, type and degree of adenomyosis might also be considered in the management of adenomyosis. Thus, in future TVS will have an important role in evaluating adenomyosis, which might require expert sonologists in centers dedicated for diagnosing this.

\section{References}

1. Naftalin J, Hoo W, Pateman K, Mavrelos D, Holland T, et al. (2012) How common is adenomyosis? Aprospective study of prevalence using transvaginal ultrasound in gynaecology clinic. Hum Reprod 27(12): 3432-3439.

2. MaheshwariA, Gurunath S, Fatima E, Bhattacharya S (2012) Adenomyosis and sub fertility: a systematic review of prevalence, diagnosis, treatment and fertility outcomes. Hum Reprod Update 18(4): 374-392.

3. Younes G, Tulndi T (2017) Effects of adenomyosis on in vitro fertilization treatment outcomes: a meta-analysis. Fertil Steril 108(3): 483-490.

4. Li X, Liu X, Guo SW (2014) Clinical profiles of 710 premenopausal women with adenomyosis who underwent hysterectomy. J Obstet Gynecol Res 40(2): 485-494.

5. Tsui KH, Lee WL, Chen CY, Sheu BC, Yen MS, et al. (2014) Medical treatment for adenomyosis and/or adenomyoma. Taiwan J Obstet Gynecol 53(4): 459-465.

6. Exacoustos C, Manganaro L, Zupi E (2014) Imaging for the evaluation of endometriosis and adenomyosis. Best Pract Res Clin Obstet Gynaecol 28(5): 655-681.

7. Pinzauti S, Lazzeri L, Tosti C, Centini G, Orlandini C, et al. (2015) Transvaginal sonographic features of diffuse adenomyosis in 18-30 yrs-old nullugravid women without endometriosis: association with symptoms. Ultrasound Obstet Gynaecol 46(6): 730-736.

8. Tellum T, Nygaard S, Skovholt EK, Qvigstad E, Lieng M (2018) Development of a clinical prediction model for diagnosing adenomyosis. Fertil Steril 110(5): 957-964.

9. Ferenczy A (1998) Pathophysiology of adenomyosis. Hum Reprod Update 4(4): 312-322.

10. Van de Boch T, De Bruijn AM, De Leeuw RA, Dueholm M, Exacoustos C, et al. (2018) A sonographic classification and reporting system for diagnosing adenomyosis. Ultrasound Obstet Gynaecol.

11. Pal K, Chung JE, Pak HJ, Jeung IC, Kim JH, et al. (2015) Usefulness of CA125 in the differential diagnosis of uterine adenomyosis and myoma. Eur J Obstet Gynaecol Reprod Biol 185: 131-135.
Submission Link:

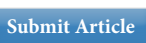

DOI: $10.32474 /$ OAJRSD.2018.02.000128

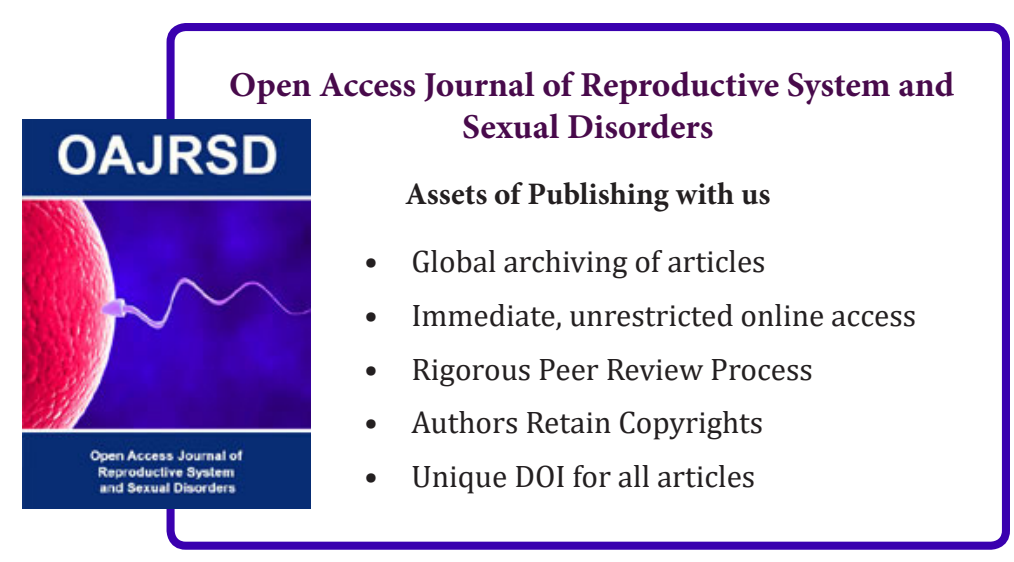

